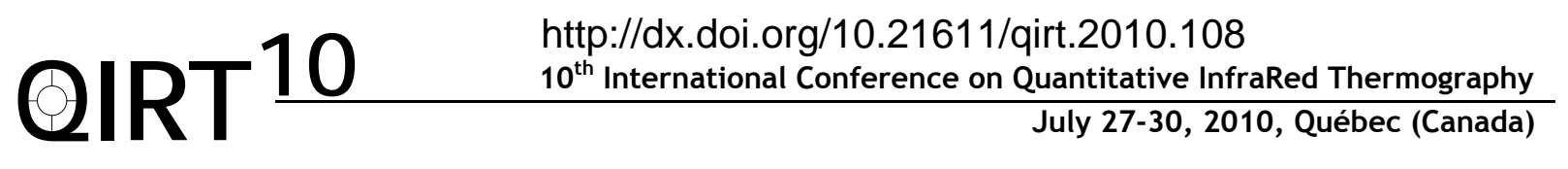

\title{
Temperature evolution in shape memory alloy during loading in various conditions
}

\author{
by E.A. Pieczyska* and H. Tobushi**
}

*Institute of Fundamental Technological Research, PAS, A. Pawinskiego 5B, 02-106 Warsaw, Poland; epiecz@ippt.gov.pl

${ }^{* *}$ Aichi Institute of Technology, 1247 Yachigusa, Yakusa-cho, Toyota, 470-0392, Japan; tobushi@aitech.ac.jp

\begin{abstract}
Mechanical characteristics and temperature changes related to stress-induced martensite transformation developing in TiNi shape memory alloy have been presented. Exothermic martensite forward and endothermic reverse transformations have been recorded with use of three kinds of infrared cameras, including very fast and sensitive Therma-Cam Fenix DTS. It was found that the temperature distribution on the surface of the specimens was uniform during straining below the austenite start temperature, while investigating shape memory effect, whereas bands of higher temperature corresponding to localized martensitic transformation were recorded during the process carried out above the alloy austenite finish temperature.
\end{abstract}

\section{Introduction}

The shape memory effect (SME) and superelasticity (SE) are the main phenomena which appear in a shape memory alloy (SMA), depending on the test temperature $T$. They are controlled by two material parameters: the austenite finish $\left(A_{f}\right)$ and the austenite start $\left(A_{S}\right)$ temperatures. If $T$ is higher than $A_{f}$, the SE appears, and if $T$ is lower than $A_{S}$, the SME appears. The behavior is caused by the stress-induced reversible martensite transformation (MT) which takes place during the SMA loading and unloading. In the case of SE, almost complete reverse transformation occurs during the SMA unloading Figs 4-9. In the case of SME, quite significant residual strain is observed after the SMA unloading (Figs 2,3). The strain related to the martensitic phase disappears, if the specimen is heated after the unloading above the $A_{f}$ temperature. The energy storage and the energy dissipation due to the SE in SMA are very large and the recoverable stress and strain are quite large compared to the traditional metals. The described properties enable the SMA many applications, e.g., as damping elements, driving force of actuators or main parts of heat engines. The MT in general can be induced by variation in temperature or stress, so the SMA behavior depends on the thermomechanical loading conditions [1-4]. The main point studied in the paper is the homogeneity of the martensite transformation process, carried out in various conditions, since the homogeneity usually assures higher reliability of the SMA applied systems.

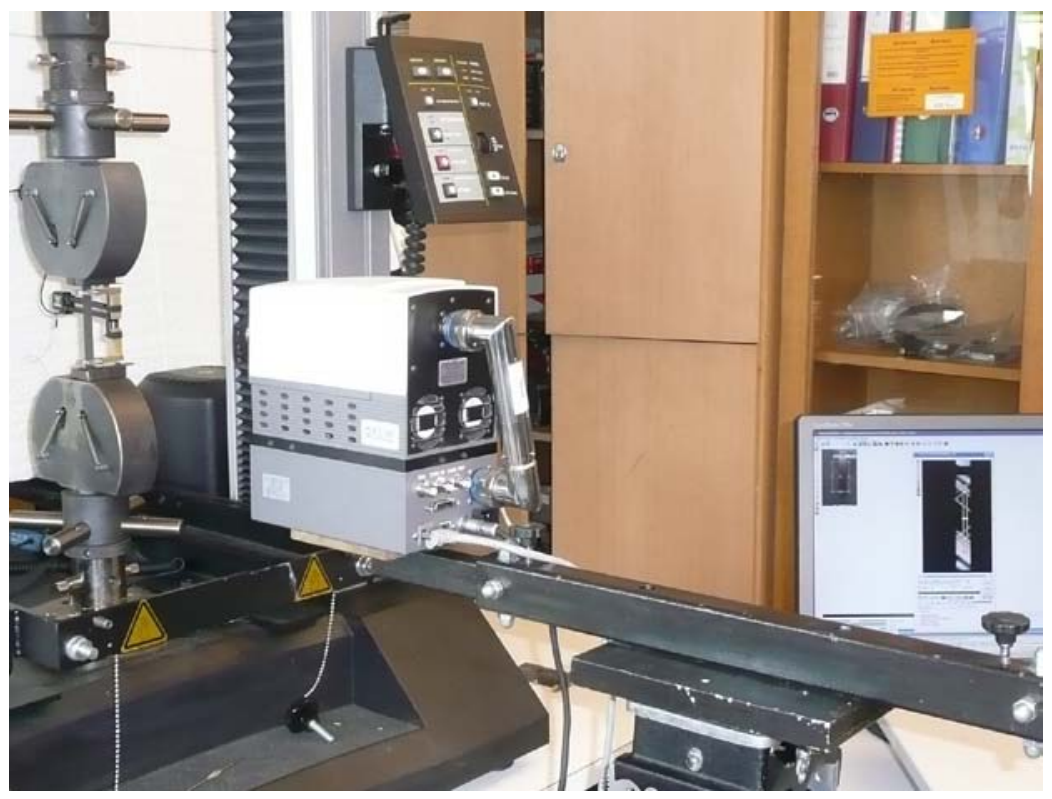

Fig. 1. Photograph of TiNi SMA specimen with mechanical extensometer in grips of Instron testing machine, Flir Phoenix infrared camera and temperature distribution image of localized martensite transformation 
The specimens have been subjected to the strain- and stress-controlled tension tests on Instron testing machine in quasi-static range of the strain rates. In the course of investigation both the mechanical characteristics and the distribution of the infrared radiation emitted by the specimen surface were registered continuously. Photograph of the experimental set-up is presented in Figure 1. TiNi SMA specimen was placed in the grips of Instron testing machine. The specimen deformation was measured by a mechanical extensometer with gauge length equal to $25 \mathrm{~mm}$. The extensometer was mounted on the thin flexible specimen by means of a specially designed grip. Before the testing, the specimen surface was covered with a very thin layer of a carbon black powder in order to make its emissivity higher and more homogeneous. The temperature measuring sensitivity was below $0.08 \mathrm{~K}$. The infrared radiation from the specimen surface was recorded with high frequency by Flir Phoenix infrared camera. One can notice in the figure the obtained image of the temperature distribution related to the localized martensite transformation (Fig. 1).

\section{Stress-strain curves and temperature distributions for shape memory alloy in tension under various conditions}

The stress-strain curves and the specimen temperature distributions obtained during tension of the TiNi SMA at temperature below, at and above the SMA austenite finish temperature $\left(A_{f}\right)$ are shown in Figs 2 - 4 . One can find from the thermograms that the temperature increases during the SMA loading and decreases during the unloading processes.

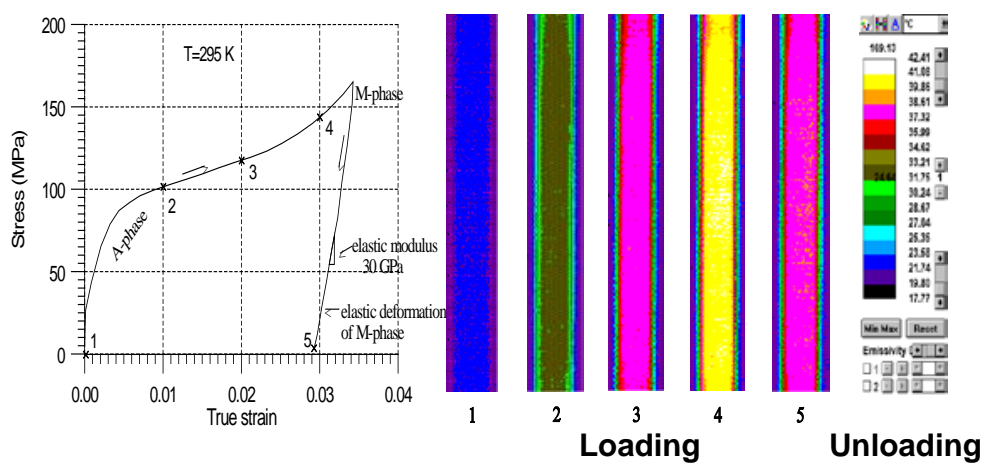

Fig. 2. Stress-strain curve and temperature distribution on the surface of TiNi SMA tape obtained at various strains under strain rate of $1.87 \times 10^{-3} \mathrm{~s}^{-1}$ during loading and unloading at $T<A_{s}$ studied by infrared thermography

The temperature distribution accompanying the deformation performed at $T<A_{f}$ presented in Fig. 2 is uniform, manifesting that the martensite forward and reverse transformation processes are homogeneous in macro-scale.
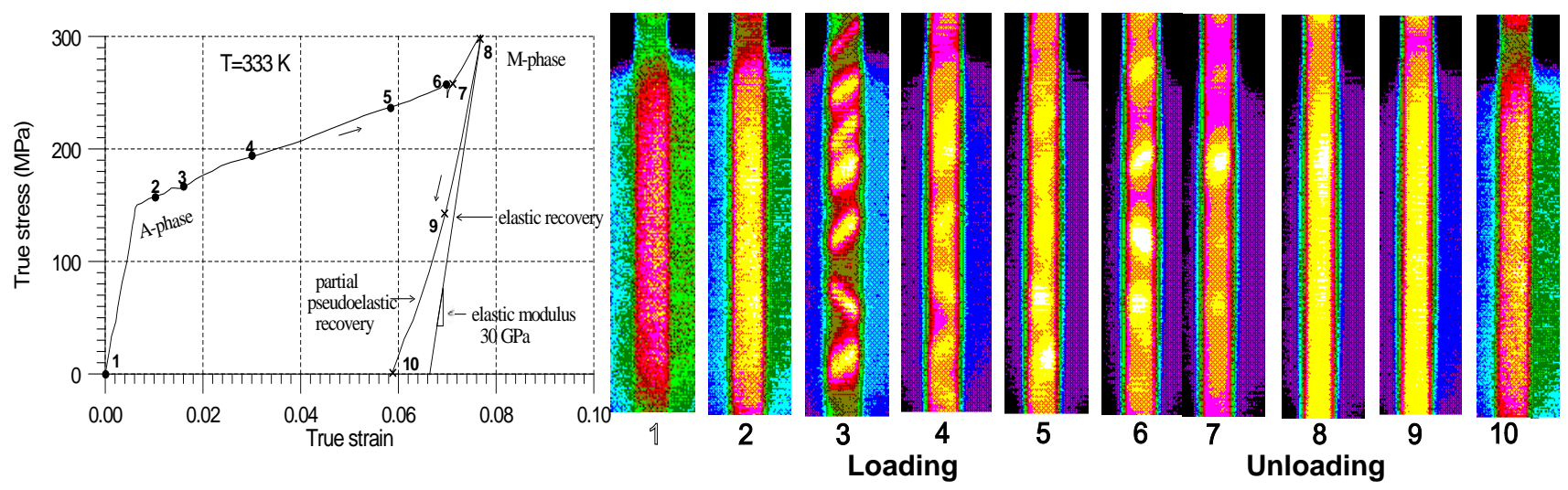

Fig. 3. Stress-strain curve and temperature distribution on the surface of TiNi SMA tape at various strains under strain rate of $1.87 \times 10^{-3} \mathrm{~s}^{-1}$ during loading and unloading at $T=A_{f}$ studied by infrared thermography

Looking at Fig. 3 one can notice that the temperature distribution of the SMA subjected to deformation at $T=A_{f}$ is not uniform, especially during the loading, manifesting that the martensite transformation process is not homogeneous. Bands of new phase, related to the higher temperatures, nucleate at various positions and grow wider thereafter on the whole surface.

One should take into account that the results presented in figures 2 and 3 were obtained for the similar sample of the shape memory alloy during tension carried out with the strain rate $1.87 \times 10^{-3} \mathrm{~s}^{-1}$. The specimen in Fig. 2 was subjected to tension in the strain range of about $4 \%$, while the strain range in Fig. 3 was about $8 \%$. 
Next results, presented in Fig. 4, were obtained for different sample of the TiNi shape memory alloy, subjected to superelastic loading within the strain range of about $6 \%$ and higher strain rate equal to $1.6 \times 10^{-2} \mathrm{~s}^{-1}$. For this SMA much higher stresses have been obtained in the range of the martensite forward transformation in comparison to the former results. After the unloading, only a small residual strain has been recorded (Fig.4, left).
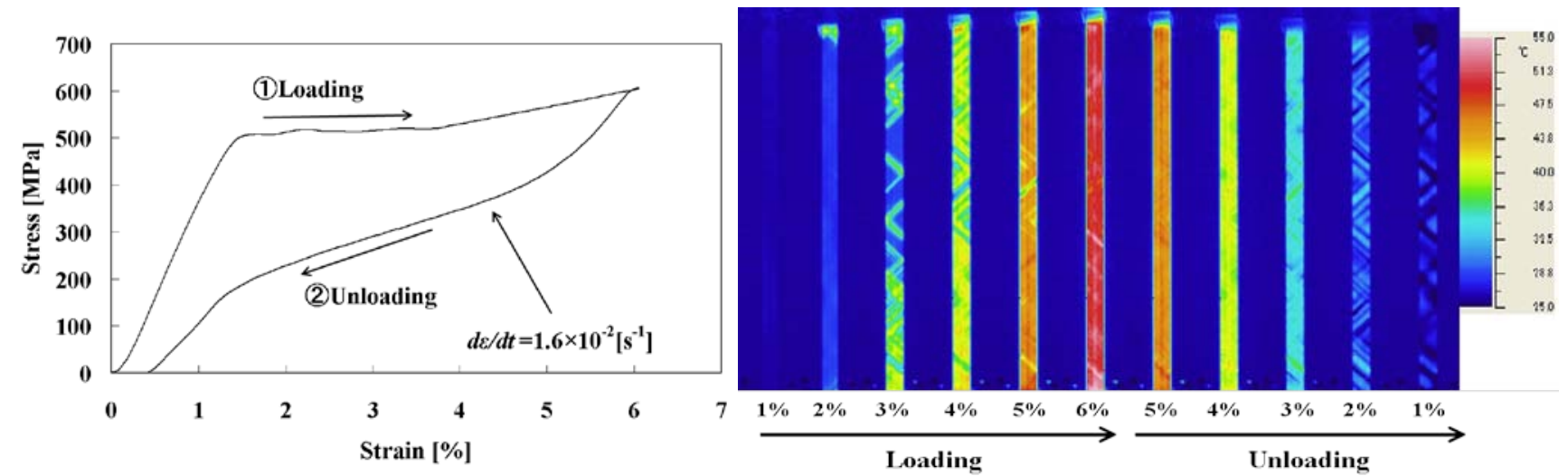

Fig. 4. Stress-strain curve and temperature distribution on the surface of a TiNi SMA tape at various strains under a strain rate of $1.6 \times 10^{-2} \mathrm{~s}^{-1}$ during loading and unloading at $T>A_{f}$ studied by infrared thermography

Looking at Fig. 4 one can observe a localized Lüders-like deformation, recorded for the tension test carried out above the $A_{f}$ temperature. Bands of significantly higher temperature, corresponding to the exothermic inhomogeneous martensite forward transformation were recorded during the SMA loading, whereas bands of significantly lower temperature, corresponding to the endothermic reverse transformation, were recorded during the SMA unloading process, respectively. It was observed that in the case of high strain rate, the transformation band nucleates not only at the ends but also at various positions and grows thereafter on the whole surface of the specimen.

Sometimes the nucleation and growth of the transformation bands due to the MT are so clear and significant that the bands on the surface of the specimen can be observed directly by naked eyes. Such effects were found during the test which results are presented in Figure 4. The photographs of the surface on the specimen are shown in Figure 5.

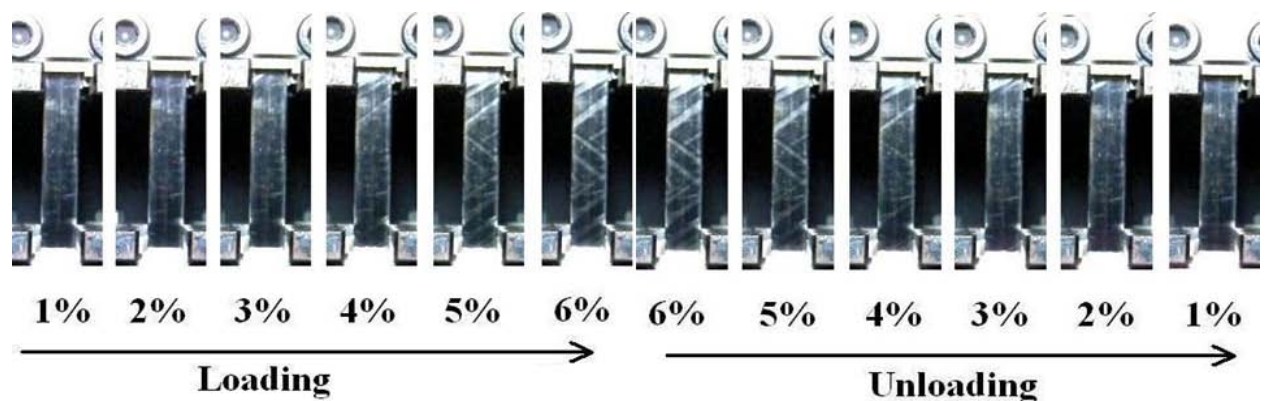

Fig. 5. Deformation patterns on specimen surface due to phase transformations in tension test under strain rate $=1.6 \times 10^{-2} \mathrm{~s}^{-1}$

\section{Thermomechanical aspects of the shape memory alloy behavior for stress-controlled tension test}

Next test has been carried out in stress-controlled conditions, with a constant stress rate equal to $25 \mathrm{MPa} / \mathrm{s}$ and a strain range of $8 \%$. In general, it is not so easy to perform such a kind of test, in particular in the case of not homogeneous specimen deformation. The mechanical characteristics shown in Figure 6 confirms that the control program of the testing machine work properly and the goal of the constant stress rate was almost achieved. Namely, a linear dependence between the stress and the time was recorded during the specimen loading and unloading. An discrepancy from the linearity has been recorded for the time range 28-36 seconds. As can be concluded from the strain vs. time curve presented in Figure 7 , at this loading stage, a very intense specimen deformation was recorded. It means that the extensometer system was not able to follow the specimen deformation with the sufficient accuracy, but probably it happened only then. The deformation was caused by the stress-induced martensite transformation, strongly intense and inhomogeneous, localized Lüders-like transformation. It starts at about $500 \mathrm{MPa}$ and develop with increasing stress till about $800 \mathrm{MPa}$, while the reverse transformation starts at about $220 \mathrm{MPa}$ and develops till the strain limit set before the test (Fig.9). 


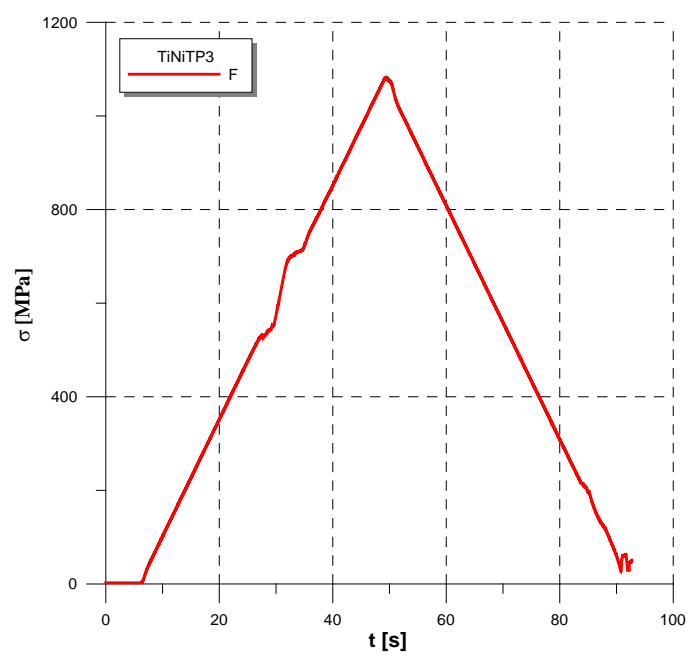

Fig. 6. Stress vs. time for TiNi SMA subjected to loading and unloading during tension test with stress rate $25 \mathrm{MPa} / \mathrm{s}$

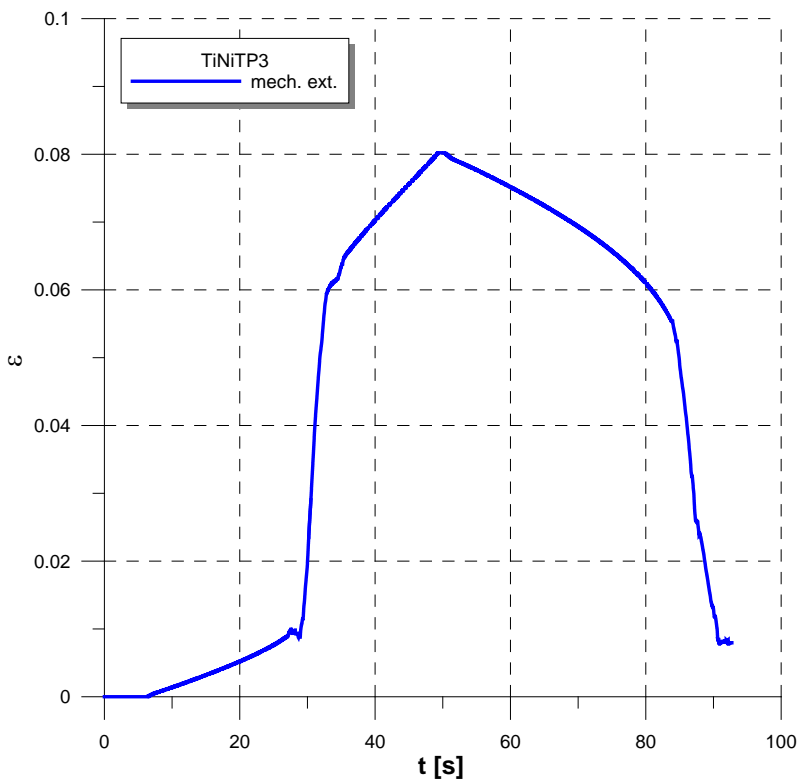

Fig. 7. Strain vs. time for TiNi SMA subjected to loading and unloading during tension test with stress rate $25 \mathrm{MPa} / \mathrm{s}$

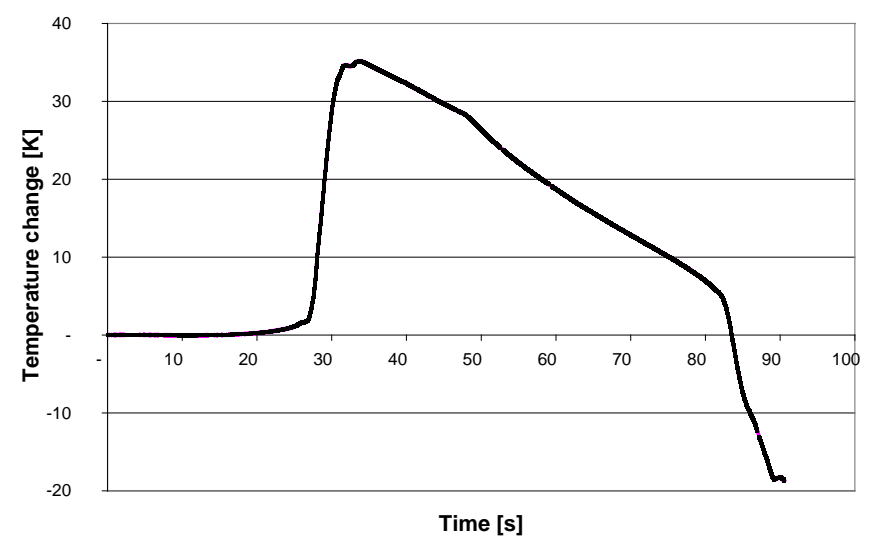

Fig. 8. Temperature change of TiNi SMA vs. time during tension test with stress rate $25 \mathrm{MPa} / \mathrm{s}$ 
The temperature changes related to the martensite transformation calculated as the average from the specimen area are presented in figure 8 . After a very fine thermoelastic effect the temperature increases till $35 \mathrm{~K}$ during the TiNi SMA loading and decreases below the initial temperature during the unloading process. The infrared technique enables us to estimate the specimen temperature changes, as well as to study the phase transformation morphology, i.e. to record the bands of significantly higher temperature due to the exothermic martensite forward and endothermic reverse transformation. Thermograms obtained at the chosen deformation stages and related to the particular points of the stress-strain curves are presented in Figure 9. One can observe bands of significantly higher temperature recorded during the SMA loading (3 - 6). At higher strains, more and more lines evolving into the bands appear started from the grip area (3) and move towards the specimen center. Finally, when the martensite transformation is completed, the temperature distribution became almost uniform again (7). During the unloading process, after passing the elastic stage, the reverse transition initiates in the central part of the specimen (8), evolving via subsequent lines of the reverse transformation characterized by significantly lower temperature (8-12). After the unloading is completed, the material almost returns to its parent austenite phase (13). The temperature drops below the initial one (Fig. 8) and the residual strains, related to a small amount of martensitic and irreversible macro-structural changes is usually recorded, depending on the specimen history; Fig.9, [6, 7].

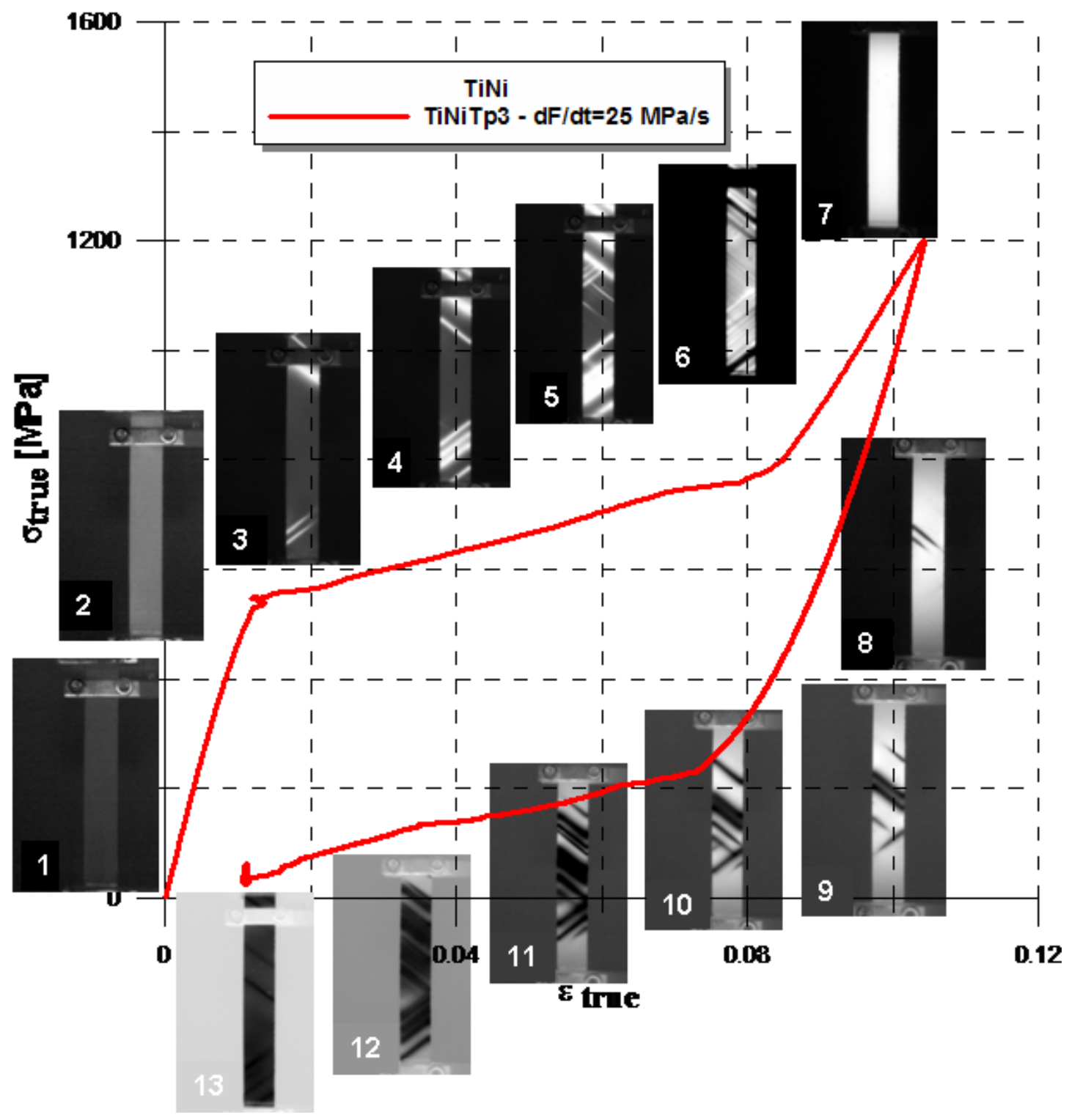

Fig. 9. Stress vs. strain curve of TiNi shape memory alloy during tension with stress rate $25 \mathrm{MPa}$ s accompanied by the specimen temperature distributions at various ranges of the martensite forward (1-7) and reverse transformation (8-13). 


\section{Discussion}

The phenomena of localized Lüders-like transformation accompanying the complete loop of TiNi SMA superelasticity behavior analyzed in infrared technique [2, 3, 5-6, 10-13], studied in modeling and simulation by Q.P. Sun research group [9], have been confirmed by Daly et al. by using a Digital Image Correlation (DIC) Strain Map technique [7].

By comparison of the results obtained for the strain- and stress-controlled tension test it was found that in both approaches the bands of localized martensite forward and reverse transformation have been observed by infrared technique [6]. Moreover, it was found recently that the localized martensite transformation is preceded by a macroscopic homogeneous transformation [7] manifested by the uniform increase in temperature; Fig. 9, thermogram (2). When the transformation is completed, the observed temperature distribution became uniform again; Fig. 9, thermogram (7) [13]. In the case of the strain controlled test the effects are so strong that it is possible to observe the transformation bands directly by naked eyes; Fig.5.

On the grounds of the characteristics obtained during the experiments: $\sigma(t), \varepsilon(t), \Delta T(t), \Delta T(\varepsilon)$ presented in Figures 6-9, and the MT thermodynamic theory [4], a current martensite fraction $z(t)$ as a function of the SMA deformation time can be estimated. The inelastic deformation $\varepsilon^{p e}(z)$ related to the martensite forward and reverse transformation can be calculated by using a formula:

$$
\varepsilon^{p e}(z)=\varepsilon(z)-\sigma(z) / E \text {, where } E \text { denotes the material young modulus value, while } \varepsilon(z) \text { and }
$$

$\sigma(z) / E$ denote the SMA specimen current and elastic deformation, presented as a function of the martensite volume fraction, respectively. The martensite volume fraction is assumed as equal zero $(z=0)$, at the beginning and equal one $(z=1)$ at the end of the martensite transformation process.

\section{Acknowledgments}

The research has been carried out with partial support of the Japan Society for Promotion of Science; Post-doc IDP04774, and by the Polish Ministry of Science and Higher Education; Grant 501220837. The assistance of S.P. Gadaj, J. Luckner and M. Maj in obtaining the experimental data is appreciated.

\section{REFERENCES}

[1] Otsuka K., Wayman C.M. (Eds.), Shape Memory Materials, Cambridge Univ. Press, Cambridge, 1998.

[2] Shaw J.A. and Kyriakides S., On the nucleation and propagation of phase transformation fronts in a TiNi Alloy, Acta Mater. 45, 2, 683-700 (1997).

[3] Gadaj S.P., Nowacki W.K. and Pieczyska E.A., Temperature evolution in deformed shape memory alloy, Infrared Physics \& Tech., 43, 151-155 (2002).

[4] Raniecki B, Lexcellent C and Tanaka K, 1992, Thermodynamic models of pseudoelastic behaviour of shape memory alloys, Arch. Mech., 44-3, pp. 261-284.

[5] Pieczyska E.A., Gadaj S.P., Nowacki W.K. and Tobushi H., Investigation of nucleation and propagation of phase transitions in TiNi SMA, QIRT Journal, 1 - 1, 117-127, 2004.

[6] Pieczyska E.A., Gadaj S.P., Nowacki W.K., Tobushi H.; Phase-transformation fronts evolution for strain- and stresscontrolled tension tests in TiNi Shape Memory Alloy, Experimental Mechanics, 46, 4, (2006), 531-542.

[7] Daly S., Ravichandran G., Bhattacharya K., Stress-induced martensitic phase transformation in thin sheets of Nitinol, California Institute of Technology, Acta Materialia, 55, 2007.

[8] Pieczyska E., Nowacki W., Sakuragi T. and Tobushi H., Superelastic deformation properties of TiNi SMA, Key Engineering Materials, Vol. 340-341 (2007), 1211-1216.

[9] Hu Z.D., Sun Q.P., Zhong Z., "Numerical simulation for stress-induced phase transformation of SMAs tube under tension", Key Engineering Materials, 340-341, 1181-1186 (2007).

[10] Pieczyska E.A, Gadaj S.P., Nowacki W.K., Luckner J. and Tobushi H., Martensite and Reverse Transformation during Simple Shear of NiTi Shape Memory Alloy, Strain, 45, 93-100, 2009.

[11] Tobushi H., Pieczyska E.A., Ejiri Y. and Sakuragi T., Thermomechanical Properties of Shape-Memory Alloy and Polymer and their Composite, Mechanics of Advanced Materials and Structures, vol. 16, pp. 236-247, 2009.

[12] Tobushi H., Pieczyska E.A., Nowacki W.K. and Sugimoto Y., SMA Thin Strip for Rotary-Driving Element, Solid State Phenomena, Vol. 154, pp. 47-52, 2009.

[13] Pieczyska E., Experimental analysis of thermomechanical properties of TiNi shape memory alloys and shape memory polyurethane, Prace IPPT/ IFTR Reports, ISBN 978-83-89687-37-1, 3/2008 (in Polish, graphs in English). 